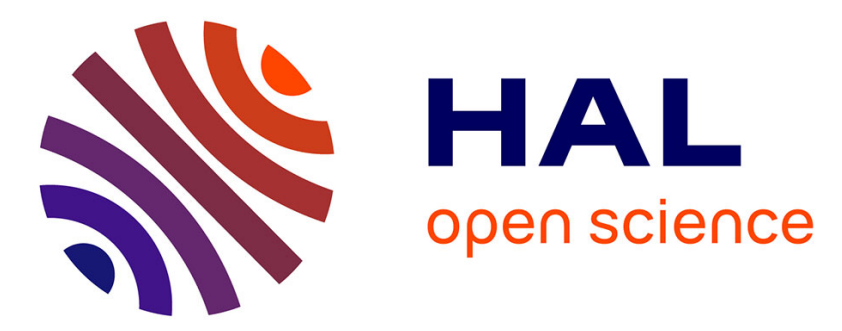

\title{
Curative pelvic exenteration for recurrent cervical carcinoma in the era of concurrent chemotherapy and radiation therapy. A systematic review
}

H. Sardain, V. Lavoue, M. Redpath, N. Bertheuil, F. Foucher, J. Leveque

\section{- To cite this version:}

H. Sardain, V. Lavoue, M. Redpath, N. Bertheuil, F. Foucher, et al.. Curative pelvic exenteration for recurrent cervical carcinoma in the era of concurrent chemotherapy and radiation therapy. A systematic review. EJSO - European Journal of Surgical Oncology, 2015, 41 (8), pp.975-985. 10.1016/j.ejso.2015.03.235 . hal-01147372

HAL Id: hal-01147372

https://hal-univ-rennes1.archives-ouvertes.fr/hal-01147372

Submitted on 18 Nov 2015

HAL is a multi-disciplinary open access archive for the deposit and dissemination of scientific research documents, whether they are published or not. The documents may come from teaching and research institutions in France or abroad, or from public or private research centers.
L'archive ouverte pluridisciplinaire HAL, est destinée au dépôt et à la diffusion de documents scientifiques de niveau recherche, publiés ou non, émanant des établissements d'enseignement et de recherche français ou étrangers, des laboratoires publics ou privés. 
Curative Pelvic Exenteration For Recurrent Cervical Carcinoma in the Era of Concurrent Chemotherapy and Radiation Therapy. A Systematic Review.

Hugo Sardain ${ }^{1,2}$, MD, Vincent Lavoue*1, 2, 3, MD, PhD, Margaret Redpath ${ }^{4}$, MD, Nicolas Bertheuil $^{2,5}$, MD, Fabrice Foucher ${ }^{1}, \mathrm{MD}$, Jean Levêque ${ }^{1,2,3}, \mathrm{MD}, \mathrm{PhD}$

${ }^{1}$ CHU de Rennes, Gynecology Department, Tertiary Surgery Center, Teaching Hospital of Rennes, Hôpital Sud, 16, Bd de Bulgarie, 35000 Rennes, FRANCE

${ }^{2}$ Université de Rennes, Faculty of Medicine, 2 Henry Guilloux, 35000 Rennes, FRANCE.

${ }^{3}$ INSERM, ER440, Oncogenesis, Stress and Signaling (OSS), Rennes, FRANCE.

${ }^{4}$ McGill University, Department of Pathology, Jewish General Hospital, Côte Sainte Catherine, Montreal (Qc), CANADA

${ }^{5}$ CHU de Rennes, Department of Plastic, Reconstructive and Aesthetic Surgery, Tertiary Surgery Center, Teaching Hospital of Rennes, Hôpital Sud, 16, Bd de Bulgarie, 35000 Rennes, FRANCE

Authors have no conflict of interest.

*Corresponding Author: Vincent Lavoue, MD, PhD, CHU de Rennes, Gynecology Department, Tertiary Surgery Center, Teaching Hospital of Rennes, Hôpital Sud, 16, Bd de Bulgarie, 35000 Rennes, FRANCE, Université de Rennes, Faculty of Medicine, 2 Henry Guilloux, 35000 Rennes, FRANCE. INSERM U1085, Team "Death Receptor \& Tumor Escape", 2 Henry Guilloux, 35000 Rennes, FRANCE. Vincent.lavoue@chu-rennes.fr 


\begin{abstract}
Objective: Pelvic exenteration requires complete resection of the tumor with negative margins to be considered a curative surgery. The purpose of this review is to assess the optimal preoperative evaluation and surgical approach in patients with recurrent cervical cancer to increase the chances of achieving a curative surgery with decreased morbidity and mortality in the era of concurrent chemoradiotherapy.
\end{abstract}

Methods: Review of English publications pertaining to cervical cancer within the last 25 years were included using PubMed and Cochrane Library searches.

Results: Modern imaging (MRI and PET-CT) does not accurately identify local extension of microscopic disease and is inadequate for preoperative planning of extent of resection. Today, only half of pelvic exenteration procedures obtain uninvolved surgical margins.

Conclusion: Clear margins are required for curative pelvic exenterations, but are poorly predictable by pre-operative assessment. More extensive surgery, i.e. the infra-elevator exenteration with vulvectomy, is a logical surgical choice to increase the rate of clear margins and to improve patient survival following surgery for recurrent cervical carcinoma. 


\section{INTRODUCTION :}

Cervical cancer represents a major public health burden with 529000 new diagnoses and 275 000 deaths annually worldwide [1]. Treatment options differ depending on the extent of tumor spread at the time of diagnosis. Early cervical cancers, defined as $\leq$ IB1 by the International Federation of Gynecology and Obstetrics (FIGO) classification [2], can be treated by surgery (radical hysterectomy and lymphadenectomy) and/or radiation therapy with equivalent results in terms of relapse-free and overall survival [3]. For cases of locally advanced cervical cancer, $\geq$ FIGO IB2, concomitant chemoradiotherapy is recommended based on the results of clinical trials from the 1990s [4-7]. Today concomitant chemoradiotherapy is the primary treatment for approximately $70 \%$ of patients [8]. Despite local control and a prolongation of disease-free survival, an estimated $20-30 \%$ of patients develop recurrent disease within the radiation field. The majority of recurrences occur 18-24 months following initial treatment. Risk of recurrence increases with FIGO stage and is estimated to be $10 \%$ for stage IB patients, $17 \%$ for IIA, $23 \%$ for IIB, $42 \%$ for III and $74 \%$ for IV [9].

When local recurrence occurs, treatment options are limited due to the frequent use of pelvic irradation for primary cervical cancer. Reirradation of the same anatomic site is contraindicated, and chemotherapy is ineffective at controlling tumors located within the previously irradiated tissue that tends to be less vascularized $[10,11]$. A recent Cochrane review was unable to compare the effectiveness of medical (radiotherapy and/or chemotherapy) versus surgical treatment for recurrent cervical cancer given the absence of randomised controlled trials [12]. Surgical resection is often the only treatment option for disease recurrence but it is associated with a high rate of complications due to the fragility of the tissue after concomitant 
radiochemotherapy [9]. Curative surgical resection of locally recurrent cervical cancer is pelvic exenteration with removal of neighboring organs such as bladder and rectum $[9,11,13]$. However, there is a lack of consensus regarding the optimal extent of the resection margins and whether the best chance of cure should include a pelvic exenteration with anterior, posterior and/or inferior exenterations. There is also no clear definition as to which patients should undergo curative versus palliative treatment. For example, lateral pelvic recurrences are considered eligible for resection by some teams, yet unresectable by others [14].

The goal of this review is to define the preoperative workup for recurret cervical cancer to guide the selection of patients for curative surgery, as well as the optimal extent of surgery in terms of morbidity and mortality. 


\section{MATERIALS AND METHODS :}

The literature was reviewed for articles published during the past 25 years using the following Medical Subject Headings (MeSH): pelvic exenteration, recurrent cervical cancer, cervical cancer treatment, radiotherapy and cervical cancer. All meta-analyses, systematic reviews and original articles written in english were reviewed. The following databases were searched:

- Medline : PubMed (Internet portal of the National Library of Medicine)

http://www.ncbi.nlm.nih.gov/sites/entrez?db=pubmed

- The Cochrane library: Cochrane-database 'Cochrane Reviews' and 'Clinical Trials' http://www3.interscience.wiley.com/cgi-bin/ mrwhome/106568753/HOME

\section{$\underline{\text { DARE }}$}




\section{$\underline{\text { RESULTS }}$}

\section{PRE-OPERATORY EVALUATION OF CERVICAL CANCER RECURRENCE :}

Evaluating the extent of recurrent tumor growth is important for proper patient management. Recurrent cervical cancer is classified as a central pelvic recurrence when the tumor is limited to the vagina, bladder, rectum and/or parametrium, and as a lateral pelvic recurrence when it spreads to the muscles and vasculature of the lateral pelvic wall. Local tumor extension needs to be accurately defined to guide proper surgical management. It is also important to eliminate the presence of metastatic tumor, which is considered to be an incurable progression of disease. Distant recurrent cervical cancer involves para-aortic, supra-clavicular or pulmonary lymph nodes in $81 \%, 7 \%$, and $21 \%$ of cases respectively [15].

Preoperative evaluation of the extent of cervical cancer spread traditionally involved clinical examination of the patient under general anaesthesia with endoscopic evaluation of the bladder and/or rectum as required. Magnetic resonance imaging (MRI) is now the preferred modality to evaluate the size of the tumor, and its relationship with neighboring organs (Table 1) [16]. Compared to computed tomography (CT), MRI has a higher sensitivity for detecting spread to the bladder (75\%), rectum (71\%), parametrium (74\%) and lymph nodes $(60 \%)$. The specificity of MRI is generally comparable to $\mathrm{CT}$, with the exception of bladder invasion which has been found to have a specificity of $91 \%$ by MRI and $73 \%$ by CT [17]. MRI can be used to predict uninvolved surgical margins with a sensitivty of $85 \%$ and a specificity of $52 \%$, with a positive predictive value of $60 \%$ and a negative predictive value of $80 \%$ [18]. Improvements in the ability of imaging to detect tumor extension will allow for more detailed preoperative planning. The surgeon will be better equipped to determine if curative surgery is feasible and will increase the chances of achieving uninvolved surgical margins. Currently all radiologic modalities are limited by their poor sensitivity in picking up microscopic disease, as well as their poor specificity in distinguishing tumor from radiation-induced fibrosis.

For the evaluation of distant metastases, positron emission tomography-computed tomography (PET-CT) is superior to MRI and CT [19]. PET-CT has been shown by Husain et al. to detect distant metastases with a sensitivity of $100 \%$ and a specificity of $73 \%$, with a positive predictive 
value of 55\% and a negative predictive value of $100 \%$ [20]. A meta-analysis of 1757 patients found that PET-CT has a sensitivity of $90 \%$ and a specificity of $99 \%$ in detecting distant metastases in cases of recurrent cervical cancer [21].

Currently the standard approach for evaluating patients with recurrent cervical cancer involves analyzing and correlating the findings from both MRI and PET-CT imaging. Combining the information provided by these two techniques allows for more precise evaluation of the size of the recurrence, the extent of invasion of adjacent structures and the presence of lymph node metastases $(p=0,041)$ [22]. Curative surgery requires complete tumor resection with uninvolved surgical margins and is a strong prognostic factor for postoperative survival. Imaging allows for better patient selection and planning of surgical procedures to increase the chances of a curative surgey, and to help avoid performing extensive surgeries, such as pelvic exenterations, for unresectable disease. In 1989 approximately 40\% of pelvic exenterations were aborted intraoperatively due to unresectable disease [23].

There is minimal data regarding the accuracy of imaging in the preoperative evaluation of lymph node involvement because metastases to pelvic lymph nodes is a contraindication for curative pelvic exenterations. Preoperative imaging has been shown to detect nodal metastases with a sensitivity of $75 \%$ and a specificity of $52 \%$, suggesting that patients should undergo lymph node dissection if there is any suspicion of involvement $[18,24]$. While there are no precise recommendations in the literature, patients without preoperative evaluation of their lymph nodes should have them removed laparoscopically and evaluated intraoperatively in all cases, even if the patient received neoadjuvant radiation.

\section{SELECTION OF PATIENTS ELLIGIBLE FOR CURATIVE SURGERY :}

When Dr. Brunschwig first described the technique of pelvic exenteration in 1948, it was considered a palliative treatment to remove gastric and/or urinary fistulas with a survival rate of less than $20 \%$ at 5 years [25]. Today the goal of pelvic exenteration is curative, aiming to achieve complete tumor resection with margins that are free of microscopic disease. According to some studies, the survival rate at 5 years is now closer to $60 \%$ with an acceptable rate of postoperative morbidity (Tables 2 and 4). In patients with visceral or lymph node metastases, there is no proven benefit of attempting curative surgery. Pelvic exenterations are therefore reserved for isolated central recurrences or lateral pelvic recurrences that do not involve the 
sciatic nerve, in patients whose general health and nutritional status permit such an extensive surgery $[14,26]$.

\section{PROGNOSTIC CRITERIA FOR ISOLATED PELVIC RECURRENCE OF CERVICAL}

\section{CANCER :}

There are several prognostic factors that should be evaluated in patients with recurrent cervical cancer, that is limited to the pelvis, before considering a pelvic exenteration:

1) Size of tumor recurrence - lesions measuring more than $5 \mathrm{~cm}$ in diameter have been shown by some authors to have almost no chance of remission despite complete removal of the tumor with uninvolved surgical margins $[9,10]$.

2) Length of time between initial cancer treatment and the recurrence - Marnitz et al found a correlation between the length of time to recurrence and patient survival such that a recurrence at less than 2 years, between 2 to 5 years and more than 5 years after initial treatment is associated with a 5-year survival rate $16.8 \%, 28.0 \%$ and $83.2 \%$ respectively [10]. Recently, Chiantera et al also determined that a recurrence occuring more than two years after initial treatment is associated with better overall patient survival rates $(p=$ 0,012) [27]. The prognostic value of time to recurrence is still debated because some authors have found no association with survival [28, 29].

3) Histologic type of recurrence- squamous cell carcinomas are associated with a significantly worse prognosis than adenocarcinomas of the cervix $(p=0,003)$. The poorer prognosis may be a reflection of the fact that perineural invasion is more frequently present in squamous cell carcinomas $(p=0,004)[30]$.

4) Presence of lymph node involvement at initial presentation - the prognostic value of lymph node involvement is controversial and has been evaluated in only a few studies. It has been found that patients presenting with lymph node metastases have a worse prognosis $[31,32]$. Recently this notion was challenged by Been et al in a study that showed no significant difference in survival rates [33].

The size of the cervical cancer recurrence, the interval of time to recurrence and the histologic type of the tumor are important elements to consider preoperatively before offering patients a 
potentially curative pelvic exenteration surgery. The age of the patient has not been found to influence overall, or disease-free, survival rates [34, 35].

Postoperatively, histopathologic evaluation of the tumor provides additional prognostic information.

1) Metastasis to mesorectal lymph nodes is significantly associated with a shorter median disease-free survival interval of 2.4 months, compared to 7.3 months in patients without mesorectal lymph node involvement $(\mathrm{p}=0,005)[27,36,37]$.

2) Lymphovascular space invasion (LVSI) is an independent prognostic factor which negatively impacted overall survival [38]. Assessing the presence of vascular emboli on pretherapeutic biopsies could facilitate the selection of patients eligible for curative pelvic exenterations [39].

3) Surgical resection margins involved by invasive carcinoma is a major significant and independent prognostic factor associated with decreased survival of patients [23]. Postoperative survival at two years drops from $55.2 \%$ with uninvolved margins to $10.2 \%$ with positive margins $(p=0,0057)$ [10]. Some authors have found that the survival rate in patients with positive margins falls to $0 \%$ after three years $[28,40]$.

In conclusion, patient survival depends on numerous pre and postoperative factors of which only one is modifiable, the achievement of surgical margins that are confirmed to be uninvolved by invasive carcinoma after histologic evaluation.

DISEASE-FREE AND OVERALL SURVIVAL IN PATIENTS FOLLOWING EXENTERATION FOR RECURRENT CERVICAL CANCER :

Since the initially reported cases of exenteration in 1948, the rate of five-year survival has increased considerably from $20 \%$ in the 1970 s to $64 \%$ in recent series where patients were better selected.

In 1989, based on the analysis of a large series of cervical cancer patients treated by pelvic exenteration, Shingelton et al estimated that the median delay to cancer recurrence was 12 months, with death typically ensuing 3 to 5 months later [23]. More recent studies, in the era of concurrent chemoradiotherapy, have found that the median time interval to recurrence is 6.1 to 7 
months $[33,36]$. The relatively short time interval to cancer recurrence highlights the importance of assessing prognostic factors preoperatively to select patients that are best suited for exenterations.

Cervical cancer recurs locally, in the pelvis or perineum, in approximately $35-60 \%$ of relapsed patients. While $20-40 \%$ of patients with recurrent disease present with distant metastases mainly involving the lungs, lymph nodes and bone. The tendancy towards local recurrence has not changed since the introduction of concomitant chemoradiotherapy. In 1999, a review of the literature by Estape et al found that the rate of local recurrence and distant metastases is $50-64 \%$ and 19\% respectively [41]. Shingleton et al did not find an association between the type of exenteration and the type of recurrence (local vs distant) [23].

In general, for patients where exenteration is the final therapeutic option, studies have found an encouraging rate of survival. However it is difficult to compare the results of the different studies since there is a large heterogeneity with respect to the type of procedure that was performed and the type of cancer that is being investigated (cervical, endometrial, vulvar or ovarian). Studies rarely focus uniquely on cervical cancer and even more rarely on cervical cancer recurrences. As shown in table 3 , anterior, posterior, total, supra or infralevator exenterations are all described in the literature but authors rarely explain how or why a given procedure was chosen. Since uninvolved surgical margins is such an important prognostic factor that is potentially modifiable, it would be useful to understand the reasons surgeons opt for one procedure over another.

\section{DIFFERENT TYPES OF PELVIC EXENTERATIONS :}

Radical pelvic exenteration was initially described by Brunschwig as a palliative procedure for central pelvic recurrences of cervical cancer. Recurrences that are limited to the cervix and/or upper vagina are amenable to a total hysterectomy with pelvic lymph node dissection. $\mathrm{He}$ defined three additional categories of local disease recurrence and suggested the following types of resection [25]:

4) Anterior: With invasion of the bladder it is recommended that the entire bladder, uterus and vagina be removed. 
5) Posterior: Invasion of the rectum is amenable to complete excision of the vagina, uterus and involved segment of bowel.

6) Total: Invasion of the bladder and rectum requires removal of bladder, vagina, uterus and rectum.

Lateral pelvic extension was considered a contraindication to surgical exenteration for a long time because it was not possible to achieve tumor-free surgical margins [28]. In 2008, Höckel et al demonstrated that patients with recurrent cervical cancer involving the pelvic side wall can benefit from a laterally extended endopelvic resection (LEER) [32]. To date, their series includes 91 patients with a overall survival rate of $61 \%$ at 5 years [14]. The LEER technique involves the resection of some, or all, of the following lateral pelvic structures : obturator internus muscle, pubococcygeus muscle, iliococcygeus muscle, coccygeus muscle, internal iliac vessels and the lumbosacral nerve plexus. The only contraindication to the LEER procedure is involvement of the sciatic nerve $[26,42]$.

Pelvic exenterations are now subclassified into type I (supralevator), type II (infralevator), and type III (infralevator with vulvectomy) exenterations based on the extent of surgical resection. The extent of tumor extension dictates the type of exenteration that is required to obtain uninvolved surgical margins. A study by Magrina et al found that there was no significant difference in patient survival between the three types of exenteration, provided that no cancer was present at the resection margins [43]. Recently, these results were confirmed by Berek et al et Yoo et al [28, 36]. Currently, a type III resection is feasible for any recurrence but is preferentially performed in patients with a recurrent lesion larger than $5 \mathrm{~cm}$ or one that has invaded the anal canal, the lower part of the vagina and/or the vulva [43].

Given the strong prognostic significance of uninvolved surgical margins, several pre and intraoperative evaluations are performed, such as preoperative imaging to select patients with resectable lesions, intraoperative exploration to ensure complete surgical removal of all macroscopically visible tumor and intraoperative evaluation of the status of surgical resection margins. Despite these measures, $7-35 \%$ of exenterations performed with a curative intent, are found to have tumor present at the surgical resection margin after thorough pathological evaluation (Table 3). To reduce this risk, a type III exenteration can be proposed to patients. With the improvement of perineal and pelvic reconstruction techniques, the rate of intra and 
postoperative complications is reasonable (Table 4) and the quality of life is comparable for all types of exenterations.

\section{MORBIDITY AND MORTALITY ASSOCIATED WITH PELVIC EXENTERATIONS :}

Pelvic exenterations are complex surgeries that are associated with a significant risk of morbidity. Resection of previously irradiated pelvic tissue leaves a large cavity that is prone to perineal leaking, poor primary wound healing and complications secondary to the obstruction of the ureters or bowel.

The rate of early postoperative complications (within 30 days of the surgery) varies from 16$71 \%$. One of the most frequent complications is gastrointestinal fistulas with connections to the skin, urinary system or vagina. Other common complications include blood clots and leaking anastomoses. There are two main factors influencing the rate of early complications: preoperative radiation-induced tissue damage and the length of the operation [44]. The rate of late postoperative complications (occuring more than 30 days after surgery) ranges from 36$61 \%$. Late complications include enterocutaneous and vaginal fistulas, ureteral obstruction, bowel obstruction and pyelonephritis. These complications arise secondary to postoperative adhesions, tumor recurrence and urinary tract infections precipitated by self-catheterization [36]. Magrina et al did not find a significant difference between the rate of early or late complications and length of hospital stay among the different types of exanterations [43]. These findings were recently confirmed by Yoo et al [36]. The mortality asscoiated with intra and postoperative complications varies from $0-12 \%$ depending on the study (Table 4). Benn et al found that survival time is significantly reduced in patients that develop postoperative complications that are considered complex $(\mathrm{p}=0,03)[33]$.

The morbidity associated with type III exanterations has been shown to be reduced in patients with pelvic floor reconstructions. The rate of bowel obstruction, pelvic abscess and fistula formation is decreased when musculocutaneous flaps are used to fill the pelvis for vaginal reconstructions $[24,44]$. Miller et al, found that filling of the pelvic cavity led to a decrease in the rate of fistula formation from 16 to $4.5 \%$ [45].

The rates of postoperative complications vary widely in the literature. Dindo et al proposed a grading system for the classification of complications as a comparison tool, but few authors have 
adopted it [46]. Certain severe complications appear to occur significantly less often when pelvic reconstruction is performed, which is an argument in favor of type III exanterations. Type III exanterations may also increase the probability of tumor-free resection margins.

\section{RECONSTRUCTION :}

Various techniques for urinary, gastrointestinal and pelvic reconstruction have been developed for the different types of pelvic exenteration to improve the quality of life of patients $[24,44]$.

\section{Urinary reconstruction :}

The technique used for urinary reconstruction depends on the preference of the surgeon and the anatomical condition of the pelvis following radiation therapy. A cutaneous ureterostomy is the least technically challenging approach, but it has become obsolete due to problems with the required equipment [47]. Currently, two techniques are performed:

- Bricker non-continent ileal conduit urinary diversion involves anastomosing the ureters to a $15-20 \mathrm{~cm}$ segment of unirradiated ileum that opens into a cutaneous stoma of the right iliac fossa

- Miami Pouch continent ileocolic urinary diversion consists of anastomosing the ureters to a low-pressure reservoir using distal ileum, right colon or proximal transverse colon. A high pressure valve at the skin's surface helps avoid incontinence. Rome, Indiana and Mainz 1 and 2 are all modified version of the Miami pouch.

The major early postoperative complications are similar for both types of urinary reconstruction, and are primarily ureteral and gastrointenstinal anastomotic leaks. Goldberg et al described a complication rate of approximately 14\% [48]. Houvenaeghel et al found that preoperative radiation increases the risk of postoperative complications and they suggest using a nonirradiated segment of bowel for the conduit to reduce this risk [47]. The main late postoperative complications are urinary tract infections and ureteral obstruction in 19 and 22\% of cases, and are more frequently associated with continent diversions [49].

There is no consensus as to which technique of urinary diversion should be performed. The Miami technique is appealing because it provides continence, but it can lead to other complications (catheterization difficulties $19 \%$ to $33 \%$ of cases $[58,59]$ ) that negatively affect 
the patient's quality of life, which is why the non-continent Bricker approach is still an important alternative.

A newer surgical technique was developed in which a segment of ileum is used to construct an orthotopic bladder, which avoids the need for a stoma and was hoped to increase the patient's quality of life. This technique has a $50 \%$ rate of continence with a complication rate of $12.5 \%$, compared to a complication rate of $2.9 \%$ for "standard" diversions [50]. This technique is rarely performed and is reserved for type I or II exenterations. So far it has not been shown to improve quality of life and thus should not be used as an argument against a type III exenteration.

\section{Gastrointestinal reconstruction :}

The standard reconstruction of rectal resections during pelvic exenterations is a definitive leftsided colostomy. Direct anastomoses are preferable in terms of quality of life, but despite advances that facilitate this procedure, such as automatic suturing devices, this technique is associated with a high rate of major complications which is thought to be related to prior pelvic irradiation. Failure of the anastomosis is the most common complication, even in patients with proximal protective stomas $[49,51]$. A recent study by Chiantera et al found that the complication rate increases from 14.5 to $20.4 \%$ when a colostomy reversal is performed [50]. In addition, Goldberg et al suggest that this technique should be avoided based on the finding that $45 \%$ of their patients suffered from early tumor recurrence at the site of colorectal anastomosis [48]. Currently there is no accepted technique for colostomy reversal in patients undergoing pelvic exenterration for recurrent cervical cancer.

\section{Vaginal and Perineal Reconstruction :}

Several different techniques have been developed for vaginal and perineal reconstruction [24, 44]. As previously discussed, pelvic reconstruction reduces the risk of postoperative morbidity by filling the cavity that is created during pelvic exenteration and lowering the incidence of gastrointestinal fistulas and obstruction. It can also improve quality of life by restoring female body image. For pelvic exenterations without perineal resections, there are two main techniques for vaginal reconstruction:

- A pedicle graft of greater omentum, using either of the gastroepiploic arteries, can be introduced into the pelvis for vaginal reconstruction. To achieve a tubular shape the 
tissue is placed around an inflatable device that is fixed to the vulvar vestibule. This is a simple surgical technique associated with a $35 \%$ risk of vaginal stenosis [52].

7) Bowel neocolpoplasty involves using a portion of the sigmoid or caecum to reconstruct the vagina. The segment of bowel that is used depends on the field of previous irradiation. The main complication of this technique is diversion colitis which can lead to frequent discharge requiring vaginal irrigation [53].

The most reliable techniques for pelvic exenterations with perineal resections involve musculocutaneous flaps that fill the pelvic cavity and allow for vaginal reconstruction when necessary. Currently, two main techniques are practiced:

8) Gracilis flaps were the first reconstruction to be described and are associated with a rate of skin paddle necrosis that varies from $14-25 \%$ depending on the study [54]. When vaginal reconstruction is desired, bilateral gracilis flaps are used [55].

9) A pedicled vertical (VRAM) and transverse (TRAM) rectus abdominis myocutaneous flap are approaches with several advantages due to the vascular reliability of the inferior epigastric artery. Houvenaeghel et al described only one case of necrosis among twenty patients that underwent the procedure [56]. Additionally, Soper et al found that only $12 \%$ of patients developed vaginal strictures or stenosis [57]. I realize you described vascular stenosis but I did not see any mention of vascular stenosis in reference 66 but they did describe a $12 \%$ rate of vaginal stenosis. A rectus myocutaneous flap is larger than a gracilis, which permits easier vaginal reconstruction using a single flap. The disadvantage of this technique is that it creates an abdominal wall weakness with $6-10 \%$ of patients developing incisional hernias [44].

10) Of note, free flaps anastomosed to iliac vessels have been described using abdominal or latissimus dorsi muscles, but this requires significant surgical experience [44].

In 1996, a study found that $95 \%$ of patients were satisfied with the vaginal reconstruction even though only $47 \%$ of them were sexually active [58]. Similarly, Goldberg et al described the rate of satisfaction and sexual activity as $85 \%$ and $56 \%$ respectively [ 48 ]. 
In general, the improvement in reconstruction techniques allows for type 3 exenterations that do not significantly alter the quality of life of patients. Unfortunately, colorectal anastomoses for gastrointestinal reconstruction are associated with a risk of morbidity that is too significant to be used regularly. Currently there is not enough evidence about patient outcome following ileal bladder reconstruction following type 3 exenterations. In contrast, pelvic reconstruction is associated with high levels of patient satisfaction [43].

\section{NEOADJUVANT AND ADJUVANT TREATMENT :}

The efficacy of neoadjuvant and adjuvant treatments are currently being evaluated to improve patient outcome.

\section{Intraoperative Radiation Therapy:}

High dose intraoperative radiation therapy was initially used when the margins of a laterally extended endopelvic resection were found to be involved with cancer by intraoperative pathologic assessment. This technique was shown to increase patient 5-year survival from 11 to $42 \%$ [59]. It was also found to cause significant gastrointestinal and nervous system toxicity in $25 \%$ and $30 \%$ of patients respectively [60].

\section{Neoadjuvant Chemotherapy:}

A recent study by Landoni et al investigated the role of preoperative chemotherapy for patients presenting with poor prognostic factors such as tumor size larger than five centimeters, lateral pelvic extention of tumor and recurrence less than a year after initial treatment. The chemotherapy was prescribed as three cycles of Taxol, Ifosfamide and Cyclophosphamide. While the study only involved 31 patients, the results were encouraging with tumor shrinkage in $61 \%$ of patients with a rate of uninvolved margins, complications, overall and disease-free survival comparable to patients who initially presented with a better prognosis [61].

Complete surgical excision remains the gold standard treatment for cervical cancer recurrence. As not all patients are eligible for curative surgery, it is hoped that the trials investigating radiation and chemotherapy will lead to improved patient care. 


\section{CONCLUSION :}

Pelvic exenteration is a complex surgical procedure that remains the sole curative therapeutic option for recurrent cervical cancer in patients that received prior radiation therapy. Preoperative patient selection requires MRI and PET-CT imaging that does not demonstrate any evidence of tumor metastasis. There is a lot of heterogeneity in the design of studies in the literature in terms of patient selection (exenteration for cervical, endometrial, vulvar and/or vaginal cancer recurrence), procedure type (anterior, posterior or total, supra or infralevator exenterations with or without perineal resection) and the recording of postoperative complications (lack of standardized classification). Two large studies from 1989 and 1997 found that the type and level of resection did not influence patient survival [23, 43]. Many large studies have found that the strongest independent and modifiable prognostic risk factor is final surgical margins that are free of cancer. Since the preoperative workup does not allow for accurate prediction of postoperative margin status, it is prudent to offer type 3 exenterations (infralevator with perineal resection) to increase the chance of uninvoled surgical margins, and thus improve patient survival, in cases of recurrent cervical cancer following pelvic radiation therapy with or without chemotherapy, especially if quality of life can be improved by using reconstructive techniques that protect against postoperative complications such as occlusion, fistulas and pelvic abscesses.

\section{BIBLIOGRAPHIE :}

[1] Ferlay J, Shin HR, Bray F, Forman D, Mathers C, Parkin DM. Estimates of worldwide burden of cancer in 2008: GLOBOCAN 2008. Int J Cancer. 2010;127:2893-917.

- [2] Delgado G, Bundy B, Zaino R, Sevin BU, Creasman WT, Major F. Prospective surgicalpathological study of disease-free interval in patients with stage IB squamous cell carcinoma of the cervix: a Gynecologic Oncology Group study. Gynecologic oncology. 1990;38:352-7. 
- [3] Landoni F, Maneo A, Colombo A, Placa F, Milani R, Perego P, et al. Randomised study of radical surgery versus radiotherapy for stage Ib-lla cervical cancer. Lancet. 1997;350:535-40.

- [4] Rose PG, Bundy BN, Watkins EB, Thigpen JT, Deppe G, Maiman MA, et al. Concurrent cisplatin-based radiotherapy and chemotherapy for locally advanced cervical cancer. $N$ Engl J Med. 1999;340:1144-53.

- $\quad$ [5] Peters WA, 3rd, Liu PY, Barrett RJ, 2nd, Stock RJ, Monk BJ, Berek JS, et al. Concurrent chemotherapy and pelvic radiation therapy compared with pelvic radiation therapy alone as adjuvant therapy after radical surgery in high-risk early-stage cancer of the cervix. J Clin Oncol. 2000;18:1606-13.

- [6] Keys HM, Bundy BN, Stehman FB, Muderspach LI, Chafe WE, Suggs CL, 3rd, et al. Cisplatin, radiation, and adjuvant hysterectomy compared with radiation and adjuvant hysterectomy for bulky stage IB cervical carcinoma. N Engl J Med. 1999;340:1154-61.

- [7] Green JA, Kirwan JM, Tierney JF, Symonds P, Fresco L, Collingwood M, et al. Survival and recurrence after concomitant chemotherapy and radiotherapy for cancer of the uterine cervix: a systematic review and meta-analysis. Lancet. 2001;358:781-6.

- [8] Gadducci A, Tana R, Cosio S, Cionini L. Treatment options in recurrent cervical cancer (Review). Oncol Lett. 2010;1:3-11.

- [9] Peiretti M, Zapardiel I, Zanagnolo V, Landoni F, Morrow CP, Maggioni A. Management of recurrent cervical cancer: a review of the literature. Surg Oncol. 2012;21:e59-66.

- $\quad$ [10] Marnitz S, Kohler C, Muller M, Behrens K, Hasenbein K, Schneider A. Indications for primary and secondary exenterations in patients with cervical cancer. Gynecologic oncology. 2006;103:1023-30.

- $\quad$ [11] Hockel M, Dornhofer N. Pelvic exenteration for gynaecological tumours: achievements and unanswered questions. Lancet Oncol. 2006;7:837-47.

- [12] Ang C, Bryant A, Barton DP, Pomel C, Naik R. Exenterative surgery for recurrent gynaecological malignancies. Cochrane Database Syst Rev. 2014;2:CD010449.

- [13] Coleman RL, Keeney ED, Freedman RS, Burke TW, Eifel PJ, Rutledge FN. Radical hysterectomy for recurrent carcinoma of the uterine cervix after radiotherapy. Gynecologic oncology. 1994;55:29-35.

[14] Hockel M, Horn LC, Einenkel J. (Laterally) extended endopelvic resection: surgical treatment of locally advanced and recurrent cancer of the uterine cervix and vagina based on ontogenetic anatomy. Gynecologic oncology. 2012;127:297-302.

- $\quad$ [15] Webb MJ, Symmonds RE. Site of recurrence of cervical cancer after radical hysterectomy. American journal of obstetrics and gynecology. 1980;138:813-7.

- $\quad$ [16] Rockall AG, Ghosh S, Alexander-Sefre F, Babar S, Younis MT, Naz S, et al. Can MRI rule out bladder and rectal invasion in cervical cancer to help select patients for limited EUA? Gynecologic oncology. 2006;101:244-9.

- [17] Bipat S, Glas AS, van der Velden J, Zwinderman AH, Bossuyt PM, Stoker J. Computed tomography and magnetic resonance imaging in staging of uterine cervical carcinoma: a systematic review. Gynecologic oncology. 2003;91:59-66.

- $\quad$ [18] Forner DM, Meyer A, Lampe B. Preoperative assessment of complete tumour resection by magnetic resonance imaging in patients undergoing pelvic exenteration. Eur J Obstet Gynecol Reprod Biol. 2010;148:182-5.

- [19] Unger JB, Ivy JJ, Connor P, Charrier A, Ramaswamy MR, Ampil FL, et al. Detection of recurrent cervical cancer by whole-body FDG PET scan in asymptomatic and symptomatic women. Gynecologic oncology. 2004;94:212-6.

[20] Husain A, Akhurst T, Larson S, Alektiar K, Barakat RR, Chi DS. A prospective study of the accuracy of 18Fluorodeoxyglucose positron emission tomography (18FDG PET) in identifying sites of metastasis prior to pelvic exenteration. Gynecologic oncology. 2007;106:177-80. 
- $\quad$ [21] Chu Y, Zheng A, Wang F, Lin W, Yang X, Han L, et al. Diagnostic value of 18F-FDG-PET or PETCT in recurrent cervical cancer: a systematic review and meta-analysis. Nuclear medicine communications. 2013.

- [22] Kitajima K, Suenaga Y, Ueno Y, Kanda T, Maeda T, Makihara N, et al. Value of fusion of PET and MRI in the detection of intra-pelvic recurrence of gynecological tumor: comparison with F-FDG contrast-enhanced PET/CT and pelvic MRI. Annals of nuclear medicine. 2013.

- [23] Shingleton HM, Soong SJ, Gelder MS, Hatch KD, Baker VV, Austin JM, Jr. Clinical and histopathologic factors predicting recurrence and survival after pelvic exenteration for cancer of the cervix. Obstetrics and gynecology. 1989;73:1027-34.

- [24] Ferron G, Pomel C, Martinez A, Narducci F, Lambaudie E, Marchal F, et al. [Pelvic exenteration: current state and perspectives]. Gynecologie, obstetrique \& fertilite. 2012;40:43-7.

- $\quad$ [25] Brunschwig A. The surgical treatment of cancer of the cervix uteri; a radical operation for cancer of the cervix. Bull N Y Acad Med. 1948;24:672-83.

- [26] Hockel M. Laterally extended endopelvic resection (LEER)--principles and practice. Gynecologic oncology. 2008;111:S13-7.

- [27] Chiantera V, Rossi M, De laco P, Koehler C, Marnitz S, Ferrandina G, et al. Survival After Curative Pelvic Exenteration for Primary or Recurrent Cervical Cancer: A Retrospective Multicentric Study of 167 Patients. International journal of gynecological cancer : official journal of the International Gynecological Cancer Society. 2014.

- [28] Berek JS, Howe C, Lagasse LD, Hacker NF. Pelvic exenteration for recurrent gynecologic malignancy: survival and morbidity analysis of the 45 -year experience at UCLA. Gynecologic oncology. 2005;99:153-9.

- [29] Sharma S, Odunsi K, Driscoll D, Lele S. Pelvic exenterations for gynecological malignancies: twenty-year experience at Roswell Park Cancer Institute. International journal of gynecological cancer : official journal of the International Gynecological Cancer Society. 2005;15:475-82.

- [30] Baiocchi G, Guimaraes GC, Faloppa CC, Kumagai LY, Oliveira RA, Begnami MD, et al. Does histologic type correlate to outcome after pelvic exenteration for cervical and vaginal cancer? Ann Surg Oncol. 2013;20:1694-700.

- [31] Wang CJ, Lai CH, Huang HJ, Hong JH, Chou HH, Huang KG, et al. Recurrent cervical carcinoma after primary radical surgery. American journal of obstetrics and gynecology. 1999;181:51824.

- $\quad$ [32] Moutardier V, Houvenaeghel G, Martino M, Lelong B, Bardou VJ, Resbeut M, et al. Surgical resection of locally recurrent cervical cancer: a single institutional 70 patient series. International journal of gynecological cancer : official journal of the International Gynecological Cancer Society. 2004;14:84651.

[33] Benn T, Brooks RA, Zhang Q, Powell MA, Thaker PH, Mutch DG, et al. Pelvic exenteration in gynecologic oncology: a single institution study over 20 years. Gynecologic oncology. 2011;122:14-8.

- [34] Matthews CM, Morris M, Burke TW, Gershenson DM, Wharton JT, Rutledge FN. Pelvic exenteration in the elderly patient. Obstetrics and gynecology. 1992;79:773-7.

- [35] Huang M, Iglesias DA, Westin SN, Fellman B, Urbauer D, Schmeler KM, et al. Pelvic exenteration: impact of age on surgical and oncologic outcomes. Gynecologic oncology. 2014;132:114-8.

- [36] Yoo HJ, Lim MC, Seo SS, Kang S, Yoo CW, Kim JY, et al. Pelvic exenteration for recurrent cervical cancer: ten-year experience at National Cancer Center in Korea. J Gynecol Oncol. 2012;23:24250.

[37] Mourton SM, Chi DS, Sonoda Y, Alektiar KM, Venkatraman ES, Barakat RR, et al. Mesorectal lymph node involvement and prognostic implications at total pelvic exenteration for gynecologic malignancies. Gynecologic oncology. 2006;100:533-6. 
- [38] Westin SN, Rallapalli V, Fellman B, Urbauer DL, Pal N, Frumovitz MM, et al. Overall survival after pelvic exenteration for gynecologic malignancy. Gynecologic oncology. 2014;134:546-51.

- [39] Sardain H, Lavoue V, Laviolle B, Henno S, Foucher F, Leveque J. Prognostic factors for curative pelvic exenterations in patients with recurrent uterine cervical or vaginal cancer. International journal of gynecological cancer : official journal of the International Gynecological Cancer Society. 2014;24:1679-85.

- $\quad$ [40] Numa F, Ogata H, Suminami Y, Tsunaga N, Nakamura Y, Tamura H, et al. Pelvic exenteration for the treatment of gynecological malignancies. Arch Gynecol Obstet. 1997;259:133-8.

- [41] Estape R, Angioli R. Surgical management of advanced and recurrent cervical cancer. Semin Surg Oncol. 1999;16:236-41.

- [42] Hockel M. Laterally extended endopelvic resection: surgical treatment of infrailiac pelvic wall recurrences of gynecologic malignancies. American journal of obstetrics and gynecology. 1999;180:306-12.

- [43] Magrina JF, Stanhope CR, Weaver AL. Pelvic exenterations: supralevator, infralevator, and with vulvectomy. Gynecologic oncology. 1997;64:130-5.

- [44] Ferron G, Martel P, Querleu D. [Vaginal reconstruction after pelvic exenteration: when and which techniques?]. Bull Cancer. 2003;90:435-40.

- $\quad$ [45] Miller B, Morris M, Gershenson DM, Levenback CL, Burke TW. Intestinal fistulae formation following pelvic exenteration: a review of the University of Texas M. D. Anderson Cancer Center experience, 1957-1990. Gynecologic oncology. 1995;56:207-10.

- [46] Dindo D, Demartines N, Clavien PA. Classification of surgical complications: a new proposal with evaluation in a cohort of 6336 patients and results of a survey. Ann Surg. 2004;240:205-13.

- [47] Houvenaeghel G, Moutardier V, Karsenty G, Bladou F, Lelong B, Buttarelli M, et al. Major complications of urinary diversion after pelvic exenteration for gynecologic malignancies: a 23-year mono-institutional experience in 124 patients. Gynecologic oncology. 2004;92:680-3.

- [48] Goldberg GL, Sukumvanich P, Einstein MH, Smith HO, Anderson PS, Fields AL. Total pelvic exenteration: the Albert Einstein College of Medicine/Montefiore Medical Center Experience (1987 to 2003). Gynecologic oncology. 2006;101:261-8.

- $\quad$ [49] Angioli R, Panici PB, Mirhashemi R, Mendez L, Cantuaria G, Basile S, et al. Continent urinary diversion and low colorectal anastomosis after pelvic exenteration. Quality of life and complication risk. Crit Rev Oncol Hematol. 2003;48:281-5.

- $\quad$ [50] Chiantera V, Rossi M, De laco P, Koehler C, Marnitz S, Fagotti A, et al. Morbidity after pelvic exenteration for gynecological malignancies: a retrospective multicentric study of 230 patients. International journal of gynecological cancer : official journal of the International Gynecological Cancer Society. 2014;24:156-64.

- [51] Husain A, Curtin J, Brown C, Chi D, Hoskins W, Poynor E, et al. Continent urinary diversion and low-rectal anastomosis in patients undergoing exenterative procedures for recurrent gynecologic malignancies. Gynecologic oncology. 2000;78:208-11.

- [52] Beemer W, Hopkins MP, Morley GW. Vaginal reconstruction in gynecologic oncology. Obstet Gynecol. 1988;72:911-4.

- [53] Hage JJ, Karim RB, Asscheman H, Bloemena E, Cuesta MA. Unfavorable long-term results of rectosigmoid neocolpopoiesis. Plast Reconstr Surg. 1995;95:842-8; discussion 9-50.

- [54] McCraw JB, Massey FM, Shanklin KD, Horton CE. Vaginal reconstruction with gracilis myocutaneous flaps. Plast Reconstr Surg. 1976;58:176-83.

[55] Lacey CG, Stern JL, Feigenbaum S, Hill EC, Braga CA. Vaginal reconstruction after exenteration with use of gracilis myocutaneous flaps: the University of California, San Francisco experience. American journal of obstetrics and gynecology. 1988;158:1278-84. 
- [56] Houvenaeghel G, Ghouti L, Moutardier V, Buttarelli M, Lelong B, Delpero JR. Rectus abdominis myocutaneous flap in radical oncopelvic surgery: a safe and useful procedure. European journal of surgical oncology : the journal of the European Society of Surgical Oncology and the British Association of Surgical Oncology. 2005;31:1185-90.

- [57] Soper JT, Havrilesky L, Secord AA, Berchuck A, Clarke-Pearson DL. Rectus abdominis myocutaneous flaps for neovaginal reconstruction after radical pelvic surgery. International journal of gynecological cancer : official journal of the International Gynecological Cancer Society. 2005;15:542-8.

- [58] Ratliff CR, Gershenson DM, Morris M, Burke TW, Levenback C, Schover LR, et al. Sexual adjustment of patients undergoing gracilis myocutaneous flap vaginal reconstruction in conjunction with pelvic exenteration. Cancer. 1996;78:2229-35.

- [59] Dubois JB. [Intraoperative radiotherapy: back to the future?]. Cancer radiotherapie : journal de la Societe francaise de radiotherapie oncologique. 2009;13:423-7.

- $\quad$ [60] Gemignani ML, Alektiar KM, Leitao M, Mychalczak B, Chi D, Venkatraman E, et al. Radical surgical resection and high-dose intraoperative radiation therapy (HDR-IORT) in patients with recurrent gynecologic cancers. International journal of radiation oncology, biology, physics. 2001;50:687-94.

- [61] Landoni F, Zanagnolo V, Rosenberg PG, Lopes A, Radice D, Bocciolone L, et al. Neoadjuvant chemotherapy prior to pelvic exenteration in patients with recurrent cervical cancer: single institution experience. Gynecologic oncology. 2013;130:69-74. 
Table 1 : Performance of MRI in detecting extent of pelvic tumor invasion and presence of nodal metastases in patients with primary and/or recurrent cervical cancer.

\begin{tabular}{|c|c|c|c|c|c|}
\hline $\begin{array}{c}\text { ORGAN } \\
\text { EVALUATED }\end{array}$ & & BLADDER & RECTUM & $\begin{array}{l}\text { LATERAL PELVIC } \\
\text { COMPARTMENT }\end{array}$ & $\begin{array}{c}\text { NODAL } \\
\text { METASTASES }\end{array}$ \\
\hline \multirow[t]{3}{*}{ Popovitch ${ }^{19}$} & Se & $67 \%$ & $67 \%$ & $80 \%$ & \\
\hline & Sp & $93 \%$ & $93 \%$ & $76 \%$ & NA \\
\hline & PPV & & & $50 \%$ & \\
\hline \multirow[t]{4}{*}{ Bipat $^{17}$} & NPV & & & $100 \%$ & \\
\hline & $\mathrm{Se}$ & $75 \%$ & $71 \%$ & & $60 \%$ \\
\hline & Sp & $91 \%$ & & NA & \\
\hline & PPV & & & & \\
\hline \multirow[t]{4}{*}{ Rockall $^{16}$} & NPV & & & & \\
\hline & Se & $100 \%$ & $100 \%$ & & \\
\hline & Sp & $88 \%$ & $91 \%$ & NA & NA \\
\hline & PPV & $100 \%$ & $100 \%$ & & \\
\hline \multirow[t]{4}{*}{ Forner $^{18}$} & NPV & $7 \%$ & $17 \%$ & & \\
\hline & Se & & & $75 \%$ & $75 \%$ \\
\hline & Sp & NA & NA & $65 \%$ & $52 \%$ \\
\hline & PPV & & & $65 \%$ & $56 \%$ \\
\hline \multirow[t]{5}{*}{ Donati $^{20}$} & NPV & & & $75 \%$ & $69 \%$ \\
\hline & Se & $87 \%$ & $75-81 \%$ & $75-87 \%$ & \\
\hline & Sp & $93-100 \%$ & $97 \%$ & $94-97 \%$ & NA \\
\hline & PPV & $91-100 \%$ & $92 \%$ & $75-87 \%$ & \\
\hline & NPV & $90 \%$ & $89-91 \%$ & $94-97 \%$ & \\
\hline
\end{tabular}

Se : sensitivity, Sp : specificity, PPV : positive predictive value, NPV : negative predictive value, NA : Not available 
Table 2 : Overview of patient survival and site of recurrence following pelvic exenteration.

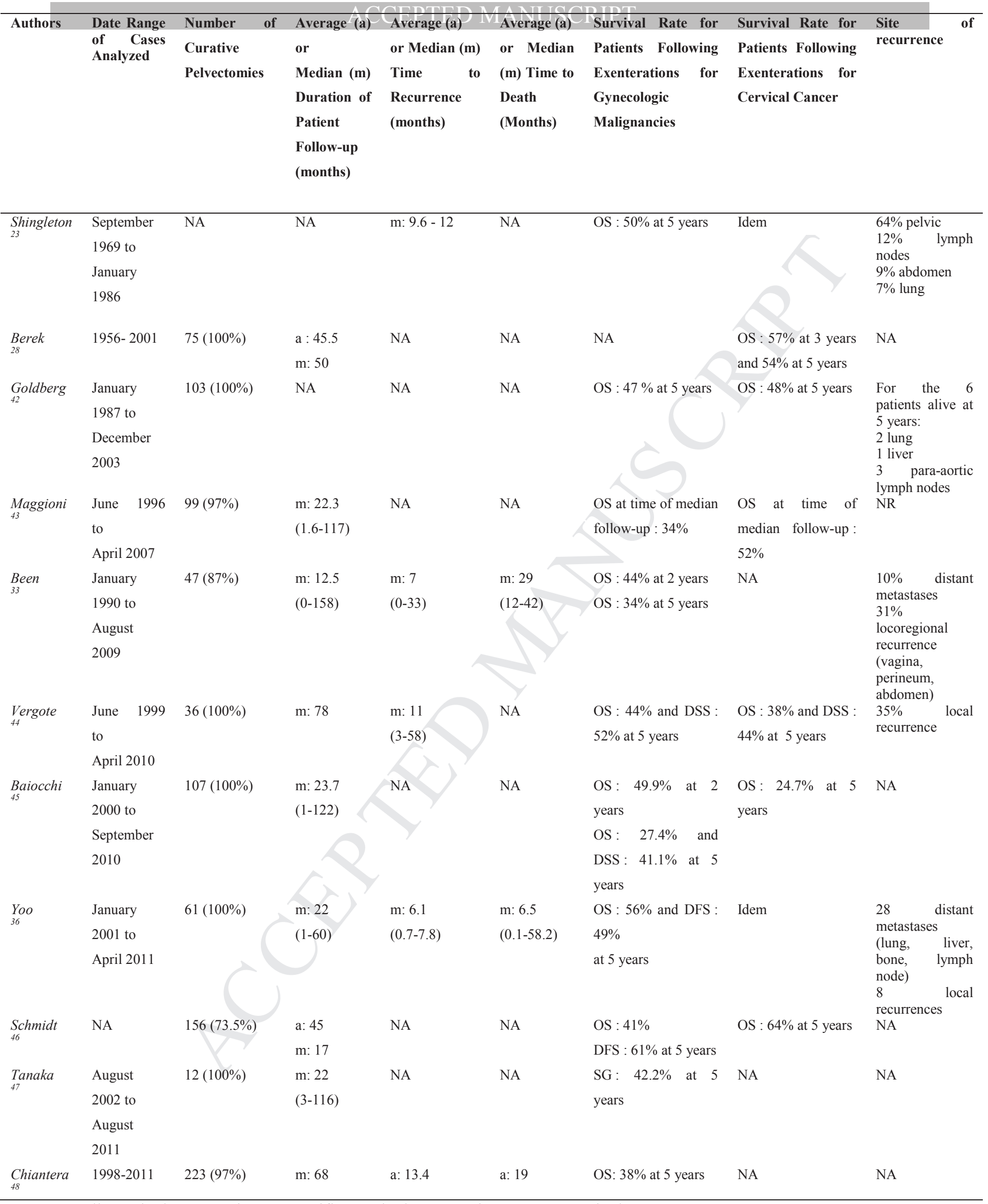

OS : Overall Survival, DSS : Disease Specific Survival, DFS : Disease Free Survival. 
Table 3 : Summary of recent studies on pelvic exenterations

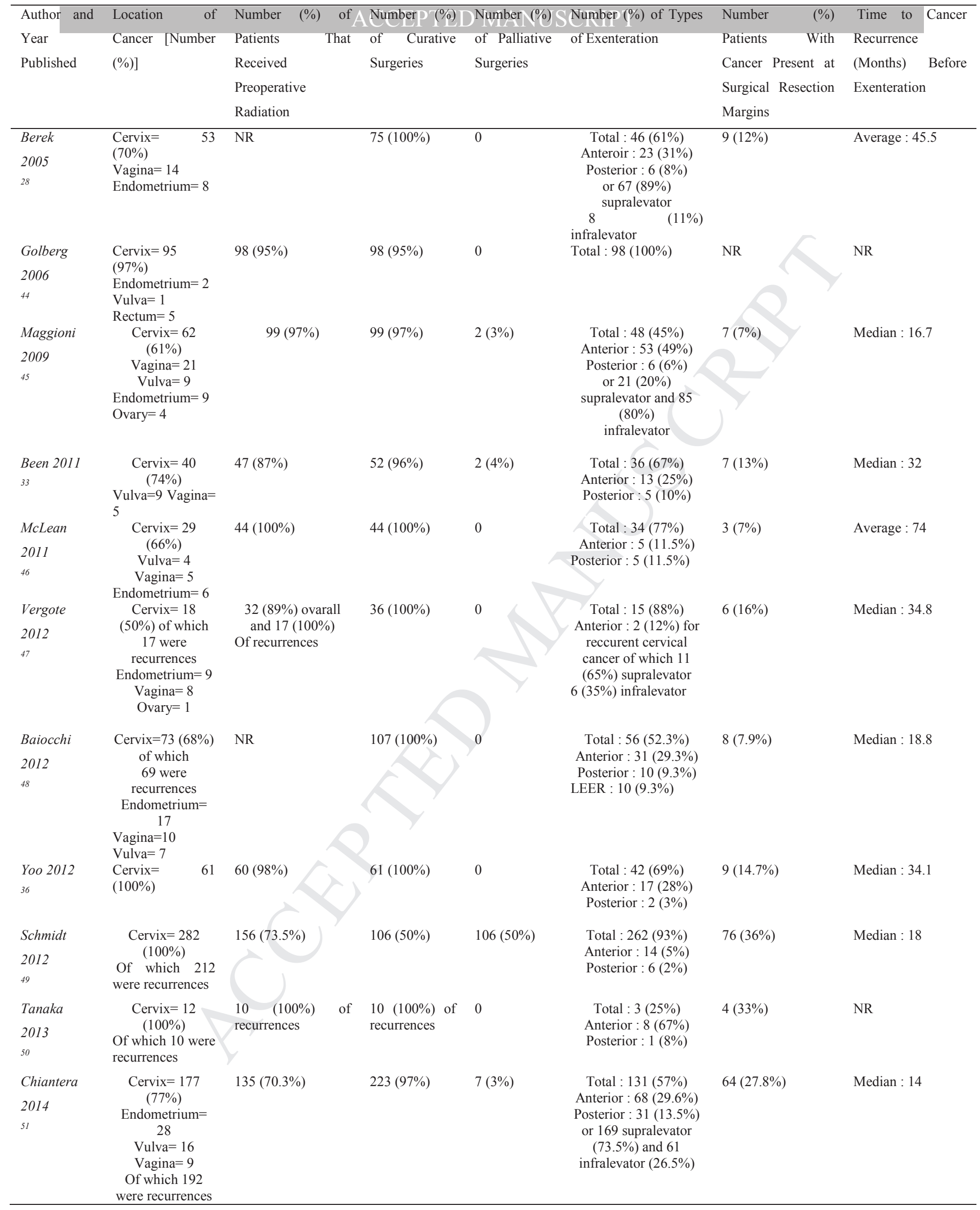


Table 4 : Overview of major postoperative complications.

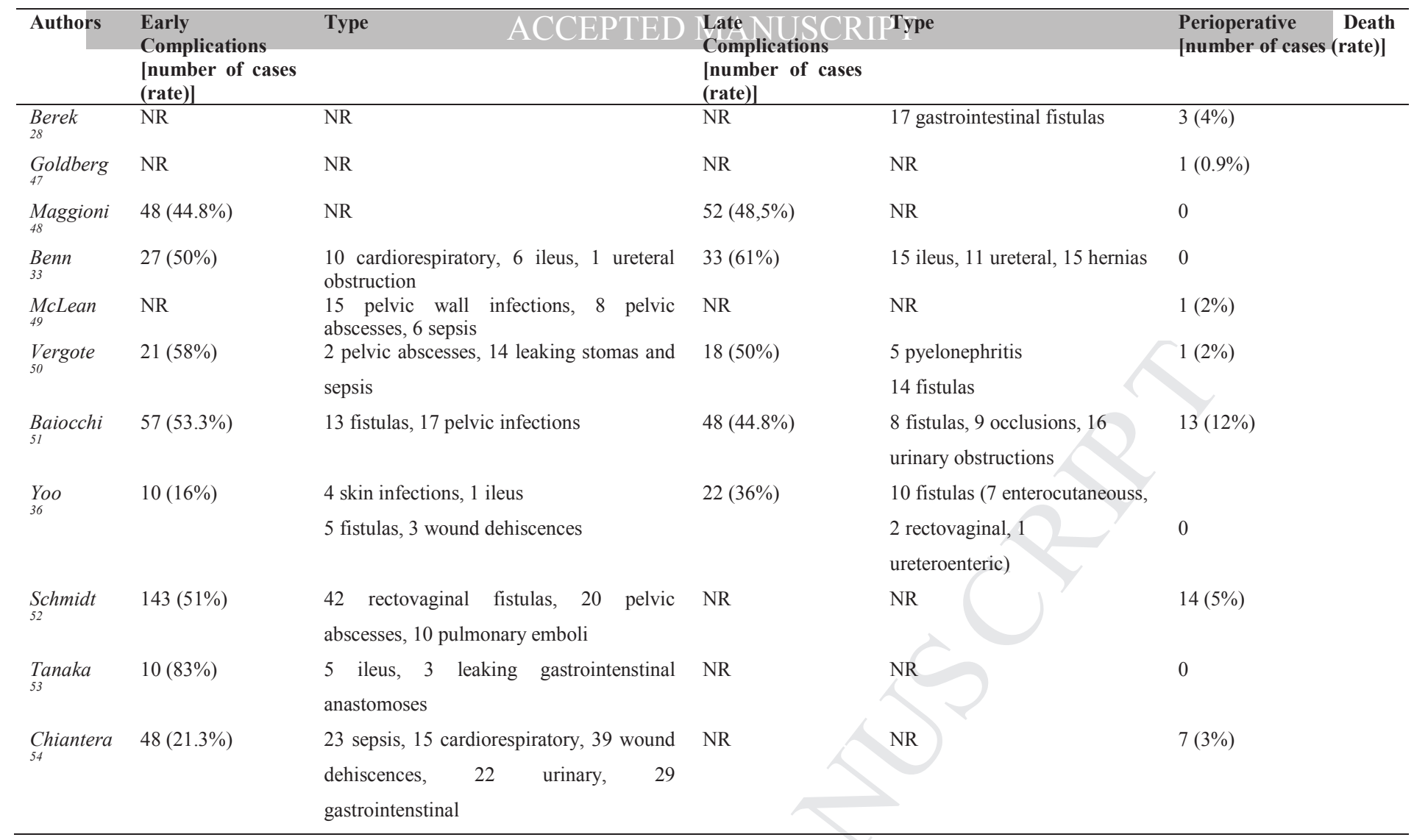

